\title{
Multi-Class Routing and Medium Access Control for Heterogeneous Mobile Ad Hoc Networks
}

\author{
Xiaojiang Du \\ Department of Computer Science \\ North Dakota State University \\ Fargo, ND 58105 \\ Xiaojiang.Du@ndsu.edu
}

\author{
Dapeng Wu, Wei Liu, Yuguang Fang \\ Dept. of Electrical \& Computer Engineering \\ University of Florida \\ Gainesville, Florida 32611 \\ \{wu, fang\}@ece.ufl.edu, liuw@ufl.edu
}

\begin{abstract}
Efficient routing is very important for Mobile Ad hoc Networks (MANETs). Most existing routing protocols consider homogeneous ad hoc networks, in which all nodes are identical, i.e., they have the same communication capabilities and characteristics. Although a homogeneous network model is simple and easy to analyze, it misses important characteristics of many realistic MANETs such as military battlefield networks. In addition, a homogeneous ad hoc network suffers from poor performance limits and scalability. In many ad hoc networks, multiple types of nodes do co-exist; and some nodes have larger transmission power, higher transmission data rate, better processing capability, and are more robust against bit errors and congestion than other nodes. Hence, a heterogeneous network model is more realistic and provides many advantages (e.g., leading to more efficient routing protocol design). In this paper, we present a new routing protocol called Multi-Class (MC) routing, which is specifically designed for heterogeneous MANETs. Moreover, we also design a new Medium Access Control (MAC) protocol for heterogeneous MANETs, which is more efficient than IEEE 802.11b. Extensive simulation results demonstrate that the MC routing has very good performance, and outperforms a popular routing protocol -- Zone Routing Protocol, in terms of reliability, scalability, route discovery latency, overhead, as well as packet delay and throughput.
\end{abstract}

\section{INTRODUCTION}

Mobile Ad hoc Networks are dynamic multi-hop wireless networks that are established by a group of mobile nodes on shared wireless channels. It is characterized by no fixed infrastructure, dynamic topologies, variable capacity links, limited physical security, bandwidth-constrained and energy constrained operation. Routing in ad hoc networks has been extensively studied over the past few years [1-8], and many ad hoc routing protocols have been proposed. Most existing routing protocols assume homogeneous MANETs, that is, all nodes in the network have the same characteristics, e.g., having the same transmission power (range), transmission data rate, processing capability, reliability, and security level. However, a homogeneous ad hoc network suffers from poor scalability, i.e., the network performance is degraded quickly as the number of nodes increases, which has been demonstrated by theoretical analyses, simulation experiments and testbed measurements [4]. Furthermore, in many realistic ad hoc networks, nodes are actually heterogeneous. For example, in a battlefield network, portable wireless devices are carried by soldiers, and more powerful and reliable communication devices are carried by vehicles, tanks, aircrafts, and satellites; these devices/nodes have different communication characteristics in terms of transmission power, data rate, processing capability, reliability, etc. So it would be more realistic to model these network elements as different types of nodes. Also there are many advantages that can be utilized to design more efficient routing protocols when nodes are modeled as different types.

In this paper, we propose a new routing protocol called Multi-Class (MC) routing, for heterogeneous MANETs. MC routing achieves good performance by exploiting node heterogeneity in many MANETs. Moreover, to further improve the performance of MC routing, we take a cross-layer approach and design a new MAC protocol called Hybrid MAC (HMAC) to cooperate with $\mathrm{MC}$ routing. Extensive simulation results demonstrate that the MC routing achieves very good performance, and outperforms a popular routing protocol Zone Routing Protocol [1], in terms of reliability, scalability, route discovery latency, overhead, as well as packet delay and throughput. In addition, our results show that MC routing is more efficient with HMAC than with IEEE 802.11b.

The rest of the paper is organized as below. Section II discusses the related work. Section III presents our MC routing protocol. In Section IV, we compare the MC routing with Zone Routing Protocol through extensive simulations. Section V concludes the paper.

\section{RELATED WORK}

We classify the related work in four aspects. First, Xu et al. [4] propose MBN routing protocol based on backbone nodes. Although our MC protocol also utilizes backbone node, MC is different from MBN in selecting backbone nodes and routing packets through backbone nodes. Second, Ye et al. [7] consider heterogeneous MANETs, and they propose to place additional reliable nodes to assist routing. In contrast, MC routing does not consider placing additional reliable nodes in the network; instead, it utilizes existing nodes in heterogeneous MANETs. Some other papers $[6,9]$ also address node heterogeneity. However, they mainly discuss how to solve the asymmetric link problem in ad hoc networks. Third, ZRP [1] is a hybrid routing protocol for MANETs that balances the tradeoff between proactive and reactive routing 
using a dynamically maintained zone topology. Differently, MC utilizes node location information to simplify routing strategy. Fourth, several routing algorithms based on location information have been proposed, such as LAR [3], DREAM [10], and GPSR [5]. However, these schemes do not utilize the heterogeneous capabilities of different nodes as we do.

\section{Multi-Class RouTing}

Many ad hoc networks are heterogeneous MANETs, where physically different nodes present. Thus it would be more realistic to model nodes in such networks as different types of nodes. For simplicity, we consider there are only two types of nodes in the network. One type of node has larger transmission range (power) and data rate, better processing capability, and is more reliable and robust than the other type. We refer to the more powerful nodes as Backbone-Capable (BC) nodes. In MC routing, BC-nodes can be elected as the Backbone nodes (B-nodes). The less powerful nodes are referred to as General nodes (G-nodes). Usually the transmission range of a B-node, denoted by $R$, is much larger than that of a general node, denoted by $r$.

The main idea of MC routing is to let most routing traffic go through B-nodes. This has the following advantages: (1) It provides better reliability and fault tolerance, since B-nodes are more reliable than general nodes. (2) It provides larger throughput since B-nodes have larger data rate than general nodes. (3) It reduces the number of hops in routing, and hence reduces the routing overhead and latency, since B-nodes have larger transmission range.

In order to utilize B-nodes in MC routing, we divide the entire routing area into multiple squares of equal size, called cells, and design a B-node selection mechanism to select a Bnode in each cell if any BC nodes exists in one cell. Under this cell structure, we design routing protocols to discover the route between any two nodes on-demand. In addition, we design a location dissemination scheme to maintain node location information used by routing. Furthermore, we propose an efficient MAC protocol that leverages the difference between B-nodes and G-nodes.

The rest of this section is organized as below. Subsection III.A presents the cell structure used by MC routing. In Subsection III.B, we describe how to select a B-node in a cell. Subsection III.C presents the MC routing protocol. In Subsection III.D, we address dissemination of node location information. In Subsection III.E, we propose a new MAC protocol.

\section{A. Cell Structure}

The routing area is divided into cells as shown in Fig. 1. Denote $a$ the side length of a cell and denote $R$ the transmission range of a B-node. If $a=R / 2 \sqrt{2}$, where $R$ is twice as much as the diagonal of a cell (see Fig. 1), then a Bnode can communicate directly with the B-node in any nearby cell. In our cell structure, one and only one B-node is elected and maintained in each cell if there are BC-nodes available in the cell. In MC routing, we assume the routing area is fixed, i.e., nodes move around in a fixed territory. This is true for many MANETs (e.g., military battlefield networks, disaster relief networks, networks in convention centers). Since the routing area is fixed, the position of each cell is also fixed. Given the location (coordinates) of a node, there is a predefined mapping between the node location and the cell, in which the node is located. To obtain this mapping, each node only needs to know the side length of a cell and the coordinates of a reference point, which serves as the origin of the entire routing area. In our architecture, a location server (to be described later) broadcasts the side length of a cell and the coordinates of a reference point to all the nodes.

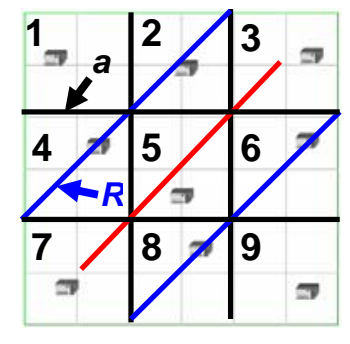

Fig. 1: Routing Cells

\section{B. B-Node Election}

Given a cell structure as described above, a B-node needs to be selected in a cell before the $\mathrm{MC}$ routing takes place. Initially, one B-node is elected in a cell if there are BC-nodes available in the cell. Since a B-node may move out of its current cell, a re-election algorithm is needed to elect a new Bnode to replace the leaving B-node. The re-election algorithm could be triggered by a leaving B-node or a G-node that notices there is no B-node in the cell due to failure of a B-node (as stated in step 6 of MC routing protocol).

The re-election algorithm works as follows. The leaving Bnode or the G-node floods an election message to all the nodes in the cell. When a BC-node receives the election message, it broadcasts a claim message that claims it will become the $\mathrm{B}$ node of the cell. Due to propagation delay, multiple BC-nodes may broadcast within a time period of not more than the propagation delay. To reduce such concurrent broadcasts, we use a random timer; i.e., each BC-node defers broadcasting its claim by a random time set by the timer. If a BC-node hears a claim message during this random time, it gives up its broadcast. Then one of the $\mathrm{BC}$-node $\mathrm{T}$ becomes the new $\mathrm{B}$ node in the cell, and $\mathrm{T}$ will start using the second address, which is the same as the cell id. Since all nodes in the cell can hear the claim message, they know that $\mathrm{T}$ is the new B-node. This idea is similar to the Random Competition based Clustering scheme in [4]. Note that those BC-nodes which do not act as B-node should act as G-nodes, e.g., using transmission range of $r$ rather than $R$.

The initial election of a B-node is similar to part of the above re-election algorithm. I.e., initially each BC-node broadcasts a claim message with a random delay, and one of the BC-node becomes the B-node. Once a B-node is elected in each cell, we can run the $\mathrm{MC}$ routing protocol, described next. 


\section{The Multi-Class Routing Protocol}

1. Each node has its own unique id; moreover, there is a unique id for each cell. In Fig. 1, the number is the id for each cell. One (and only one) B-node is elected and maintained in each cell, and each B-node has a second address, which is the same as the id of the cell in which it stays. Thus a B-node can send packet to a B-node in a nearby cell by using the second address, even though the identity of that B-node may change. 2. When a B-node moves out of a cell, it initiates a B-node election process in the cell before it leaves the current cell, and a BC-node will be elected as the new B-node using the aforementioned B-node selection scheme.

3. Routing among B-nodes. First we discuss how B-nodes find routes to other B-nodes. B-nodes use their second addresses to communicate with each other. Assume B-node $B_{S}$ (in cell $C_{S}$ ) wants to send a packet to B-node $B_{d}$ (in cell $C_{d}$ ). Although nodes move around, the cells are fixed. $B_{S}$ knows the cell in which $B_{d}$ stays based on $B_{d}$ 's second address, which is the same as the cell id. A straight line $\mathrm{L}$ is drawn between the centers of cell $C_{S}$ and $C_{d}$. An example is

given in Fig. 1, assume B-node 7 wants to send a packet to Bnode 3. The center line is line L. Two border lines which parallel to line $\mathrm{L}$ with distance of $\mathrm{W}$ from $\mathrm{L}$ are drawn from $C_{S}$ to $C_{d}$. All the cells that are within the two border lines are defined as routing cells. The value of $\mathrm{W}$ depends on the density of BC-nodes in the network. If there are enough BCnodes in the network, i.e., with high probability there is at least one BC-node in each cell, then $\mathrm{W}$ can be set as zero, i.e., routing cells are only the cells that intercept with line $\mathrm{L}$ - cell 7, 5, 3 in the example. Then the B-nodes from the routing cells form a route for $B_{S}$ to $B_{d}$. In Fig. 1, the route is $B_{7} \rightarrow B_{5} \rightarrow B_{3}$ when $\mathrm{W}$ is zero. For the simulations presented in Section IV, W is zero. If the number of BC-nodes is small, large $\mathrm{W}$ can be used. For the example in Fig. 1, if W is set as $a \sqrt{2} / 2$, where $a$ is the side length of a cell, then the routing cells are cell 7, 5, 3; 4, 2; and 8, 6. When $\mathrm{W}$ is larger than zero, routing among B-nodes is described in next step.

4. Route Discovery. Consider a node S wants to send a data packet to a destination node D. We first discuss the case where $\mathrm{S}$ is a B-node. And we will discuss the routing scheme when $\mathrm{S}$ is a general node in step 8. S is the starting B-node in routing. In $\mathrm{MC}$ routing, the starting $\mathrm{B}$-node $\mathrm{S}$ needs to know the current location of the destination node $\mathrm{D}$. The scheme by which S obtains D's location is described in step $6 \& 7$. With D's location information, $\mathrm{S}$ knows the cell $C_{d}$ in which $\mathrm{D}$ stays, and $\mathrm{S}$ knows the B-node in cell $C_{d}$ is $B_{d}$. Then $\mathrm{S}$ determines the routing cells between $\mathrm{S}$ and $B_{d}$, and sends Route Request (RR) packets to B-nodes in routing cells. If $\mathrm{W}$ is zero, then the RR packet is just forwarded by a serial of Bnode in the routing cells, e.g., $B_{7} \rightarrow B_{5} \rightarrow B_{3}$ as in the example. If $\mathrm{W}$ is larger than zero, the route is discovered as follows. The RR packet includes the following fields: Starting_B-node, Next_cells, Routing_cells, Path and
Destination_cell. Starting_B-node is the B-node initiates the route discovery. Next_cells are the cells to which current Bnode needs to multicast the RR packet. E.g, if $\mathrm{W}=a \sqrt{2} / 2$, the Next_cells for node B7 is cell 4, 5, and 8. And one multicast can be realized by a single transmission. Routing_cells are the routing cells. Path is the route via which the $\mathrm{RR}$ packet traveled, and Destination_cell is the cell including the destination node. When a B-node in routing cells receives a RR packet, it adds itself to the Path, and determines Next_cells based on the location of itself, the location of Destination_cell, and Routing_cells. The neighbor routing cells in the direction towards the destination are Next_cells. Then the B-node multicasts the RR packet to B-nodes in Next_cells. When $B_{d}$ receives the first RR packet, a route reply (RP) packet (with the discovered Path) is sent back via the coming path. And a route based on B-nodes is discovered. All other RR packets are discarded by $B_{d}$. Then $\mathrm{S}$ can start sending data packet to $B_{d}$. If S does not receive a RP from $B_{d}$ for a certain time, $S$ assumes the route discovery failed, and $S$ will flood the RR packet to all B-nodes in the network, and finds a B-node-based route to $B_{d}$. B-node flooding is needed only when the number of B-nodes in the network is small. And flooding among a small number of B-nodes does not cause large routing overhead. Assume nodes in ad hoc networks do not move very fast. (If nodes move too fast, then besides flooding data packet, no routing protocols can successfully deliver data. Since a previously discovered route will become broken because some nodes in the route move away when data packet comes.) So most of the time, destination node $\mathrm{D}$ is still in cell $C_{d}$, or in nearby cells of $C_{d}$. And since B-node $B_{d}$ can reach all nodes in nearby cells, $B_{d}$ can send the data packet to $\mathrm{D}$ directly. When $\mathrm{D}$ receives the packet, D will send an Ack (acknowledge) packet back to node $B_{d}$. If $\mathrm{D}$ is a general node, there is a unidirectional link problem here. $\mathrm{D}$ can not send the Ack packet to $B_{d}$ in one hop. Instead, the Ack packet is sent to $B_{d}$ via flooding in cell $C_{d}$ (or plus a nearby cell). If $B_{d}$ does not receive the Ack for a certain time, it means $\mathrm{D}$ is no long in the nearby cells of $C_{d}$. Node $B_{d}$ will hold the data packet (before receiving Ack) and request the updated location information of node $\mathrm{D}$ (described in step $6 \& 7$ ). Then $B_{d}$ can send the data packet to the Bnode closest to the new location of $\mathrm{D}$, and that B-node can send the packet to $\mathrm{D}$ directly. After receiving the Ack packet from destination node $\mathrm{D}, B_{d}$ will send an Ack packet to source node $\mathrm{S}$ via the incoming path.

5. Route Repair. We will use the example in Fig. 1 to discuss the route repair in MC routing. Assume the discovered route is $B_{7} \rightarrow B_{5} \rightarrow B_{3}$, when a B-node (e.g., $B_{5}$ ) in the route moves out of its cell (or fails) and no new B-node is elected, an established route becomes broken. The upstream B-node $B_{7}$ (closer to source) can detect there is no B-node in next routing cell if it does not overhear transmission from a B-node 
in the cell for a certain time after it sends the packet to the cell. Then $B_{7}$ first broadcasts route repair (RE) packets to nearby $\mathrm{B}$-nodes and tries to find a repaired route via B-nodes. The RE packet includes the next B-node $B_{3}$ and a Time-To-Live (TTL) in hops. When a B-node receives a RE packet, the TTL is decreased by 1 . And if the TTL is 0 , the B-node will not forward the RE packet. When the next B-node $B_{3}$ receives the RE packet, a Repaired packet is sent back to the B-node starting the repair via the reverse path, and an alternative path is found. If no Repaired packet is received within a timeout, the upstream B-node $B_{7}$ assumes route repair via B-nodes failed, and it will try to find a repaired route via general nodes. $B_{7}$ floods route repair (RE) packets to general nodes in the next two cells (cell 5 and cell 3 as in the example), to find out a path via general nodes to next B-node ( $B_{3}$ in the example).

The RE packet includes the following fields: Repairing_Bnode, Next_cell, Routing_cells, Destination_cell, Destination_ node and Path. Repairing_B-node is the B-node starting the route repair process. Next_cell is (cell 3 in the example) the next downstream routing cell after the cell without B-node (different from Next_cells in step 4). Routing_cells are the routing cells from Repairing_B-node to destination node. Path is the route via which the RE packet traveled, which includes both B-nodes and general nodes. An algorithm similar to AODV can be used to find a repaired route via general nodes to next B-node $B_{3}$. However, the flooding of RE packet is only in the next two routing cells (cell 5 and cell 3 in the example). When the next B-node receives the first RE packet, a route reply (RP) packet is sent back to the Repairing_B-node $B_{7}$ via the coming path. And a repaired route is discovered. All other RE packets are discarded by the next B-node $B_{3}$. Then the data packet is sent to the next B-node $B_{3}$. If general nodes in Next_cell (cell 3 in the example) do not overhead a $\mathrm{RP}$ packet from a B-node for a certain time, it means there is also no B-node in Next_cell, then general nodes in Next_cell will forward the RE packet to the next downstream routing cell (downstream cell of cell 3 in the example) according to Routing_cells field, until a B-node responses with RP packet or the destination node responses with RP packet when it receives a $\mathrm{RE}$ packet. In the worst case, i.e., if none of the routing cells have B-nodes, MC routing is similar to AODV, but the flooding area is limited to the routing cells. So even in the worst case, MC still has less routing overhead than AODV.

6. Routing from General Node. If $S$ is not a B-node, then $S$ first needs to find out a path to a nearby B-node. S floods a Route Request (RR) packet in its cell to find the nearest Bnode. $\mathrm{S}$ includes its location information in the RR packet. Only nodes in the same cell as S will process and forward the RR packet to other nodes. This reduces the overhead from flooding. A node can determine if it is in the same cell as $\mathrm{S}$ based on the location of $\mathrm{S}$ and itself. The intermediate node records its identity in the path field of the RR packet. When the B-node $B_{S}$ in the cell receives the RR packet, because $B_{S}$ has large transmission range, it can send the Route Reply (RP) packet directly to node $S$ with the path included. Then $S$ can send the data packet to $B_{S}$ following the path. And the rest is the same as in step 4. A node can discover there is no B-node in the cell if it does not receive the RP packet for a certain time after it sends out RR packet, and it will initiate a B-node election process. The B-node election process is discussed in the following.

\section{Dissemination of Node Location Information}

As mentioned in step 4, in MC routing, a start-B-node S needs to know the current location of destination node $\mathrm{D}$. Since nodes move around, an algorithm is needed to disseminate updated node location information. In this paper, we propose an efficient dissemination scheme, and it is described in the following. If a node moves within the same cell, there is no need to update its location information. When a node moves out of its previous cell, it sends a location update packet (with its new location) to the B-node in the new cell (or the nearest B-node). The location update packet can be sent out via broadcast within a small hop count. And all Bnodes periodically send aggregated node location information to a special B-node $B_{0}$. For example, $B_{0}$ can be the commands headquarter in a battlefield. The period of updating location information should not be too large, because this will cause the location information not accurate. Also the period should not be too small, because updating the location information too often will cause large overhead. The special B-node $B_{0}$ is preferred to be a fixed B-node, or a B-node only moves within one cell. If $B_{0}$ is fixed or within one cell, the dissemination algorithm is very simple. When a starting B-node $\mathrm{S}$ needs to know the location of a node $D, S$ sends a location request packet to $B_{0}$, then $B_{0}$ sends the location of D to S. Since both $S$ and $B_{0}$ are B-nodes, they know how to communicate with each other (step 2). If $B_{0}$ also moves around, then $B_{0}$ needs to multicast its current location to all B-nodes when it moves from one cell to another. Then all B-nodes know the current location of $B_{0}$, and they can request location information from $B_{0}$. In many MANETs, it is possible to choose a static or slowly-moving B-node to be $B_{0}$. And in many (like military) MANETs, it is possible and worth to deploy a static B-node as $B_{0}$. For MANETs where it is not easy to find a good candidate like $B_{0}$, the node location information dissemination can be achieved via several existing location service schemes such as [11].

\section{E. The New Media Access Control Protocol}

IEEE 802.11b is not an efficient Medium Access Control protocol. For MC routing with backbone nodes, we designed a new MAC layer protocol which is more efficient than IEEE 802.11b for heterogeneous MANETs. The key idea is to combine time-slotted mechanism with contention based mechanism. We call this new MAC protocol as Hybrid MAC 
(HMAC). In the following, we use B and G to represent Bnode and general node respectively. A large time frame is divided into 3 sub-frames which include G-to-G, B-to-B and B-to-G. G-to-G is a time slot assigned for the traffic between two general nodes. B-to-B and B-to-G time slots have similar meaning. The G-to-B traffic is included in G-to-G sub-frame, so there is no G-to-B sub-frame. Inside each sub-frame, the corresponding nodes use contention-based mechanism - IEEE 802.11b to decide which node should transmit packets. In Bto-B sub-frame, a B-node may piggyback another B-node the scheduling information in B-to-G sub-frame. An important parameter is the length of each sub-frame. The sub-frame length depends on the amount of traffic of each sub-type (e.g., G-to-G, B-to-B), which depends on many factors, including the number of B-nodes in the network, the number of general nodes in the network, the average distance between a general node and a B-node, and so on.

One can first use the estimated length of each sub-frame, and then use a scheme to adaptively adjust the sub-frame length of each type of traffic such that both fairness and good throughput are achieved. The HMAC protocol is designed to minimize contention (i.e., maximize energy efficiency) and trade off fairness with throughput.

\section{PERFormance EVAluation}

MC routing protocol is implemented in QualNet [8], a scalable packet-level simulator with an accurate radio model. HMAC was used as the MAC protocol. For the simulations presented in this Section, there are totally 20 time-slots, in which G-to-G sub-frame takes 8 time-slots, B-to-B sub-frame takes 8 time-slots, and B-to-G sub-frame takes 4 time-slots. In the following simulations, the slot allocation was fixed. We did not use any adaptive scheme to adjust the sub-frame length in the current experiments. We performed simulations with several topologies. Here, we present simulations performed by distributing 100 nodes uniformly at random in an area of $1000 \mathrm{~m} \times 1000 \mathrm{~m}$. Among the 100 nodes, 25 nodes are Backbone-Capable (BC) nodes. The transmission range of Bnode and general node are $400 \mathrm{~m}$ and $100 \mathrm{~m}$ respectively. The interference range and sensing range of a general node and a $\mathrm{BC}$-node are twice as the corresponding transmission range. The side length of a cell is set as $a=R / 1.6$. There are 16 cells in the routing area, so there will be at most 16 B-nodes. The number of B-nodes is small. The width of the routing cells -$\mathrm{W}$ is set to zero, i.e., routing cells are the cells which intercept with line L. Each simulation was run for 600 simulated seconds. The mobility in the environment was simulated using the random-waypoint mobility model. In our simulations, the pause time was set to zero seconds, which corresponds to constant motion. We control the node mobility by varying the node velocities range. The maximum velocities vary from 0 $\mathrm{m} / \mathrm{s}$ to $50 \mathrm{~m} / \mathrm{s}$. In all simulations, B-nodes have the same set of parameters in random-waypoint mobility model as general nodes.

We set the application in the following scenario. There are several source-destination pairs. The Constant Bit Rate (CBR) generators generate data traffic and sent to the destination nodes. The source sent packet of 512 bytes at a rate of 4 packets per second. We ran each simulation 10 times to get an average result for each simulation configuration. We compared our MC routing with Zone Routing Protocol (ZRP) [1], an efficient hybrid routing protocol for MANETs. IEEE 802.11 is used as the MAC protocol for ZRP. The following metrics are used to compare the routing performance.

1. Routing overhead and energy consumption. Subsection IV.A and IV.B present the results of routing overhead comparison. The result of energy consumption comparison is similar to that of routing overhead, and it is not reported in this paper because of the page limit. In subsection IV.G, we also compare the routing overhead of $\mathrm{MC}$ and $\mathrm{ZRP}$ for different densities of BC-nodes.

2. Throughput and delay. Subsection IV.C and IV.D present the results of throughput and delay comparison.

3. Scalability. The results of scalability test are reported in subsection IV.E.

In the following tests, the routing overhead of MC includes the overhead of disseminating node location information.

\section{A. Routing Overhead under Different Mobility}

First we compared the routing performance of MC with ZRP for different node mobility. The traffic load in the experiments is $150 \mathrm{kbits} / \mathrm{sec}$. Fig. 2 shows that the routing overhead of both MC and ZRP increases as the node speed increases. Higher mobility causes more broken links, and thus increases routing overhead. However, the overhead of MC is much smaller than ZRP. Since B-nodes have large transmission range, the number of hops in MC is small, and the routing latency in $\mathrm{MC}$ is low. Low routing latency means the time between route discovery and sending data packet is small, and the intermediate nodes are not far away from their previous locations when the data packet comes, which means less broken links. Under high node mobility, MC has much less broken links than ZRP, thus much less routing overhead than ZRP.

\section{B. Routing Overhead vs. Transmission Range}

We then compared the routing overhead of MC and ZRP for different node transmission range. The result is presented in Fig. 3. Here we only change the transmission range of general nodes. In the experiments, the maximum node speed is $20 \mathrm{~m} / \mathrm{s}$, and the traffic load is $150 \mathrm{kbits} / \mathrm{sec}$. Fig. 3 shows that the overhead of MC routing is much smaller than that of ZRP. This is because when the transmission range of general nodes is large enough, source node and destination node can communicate with nearby B-node directly. So there is no need to flood route request packet or Ack packet to nearby B-node, hence reduces the routing overhead a lot. 


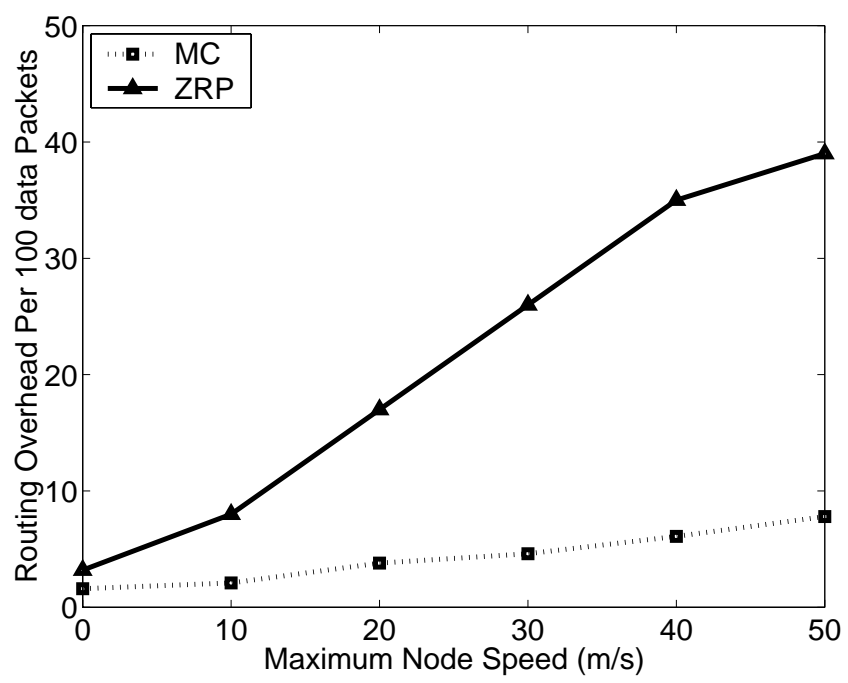

Fig. 2: Overhead under Different Mobility

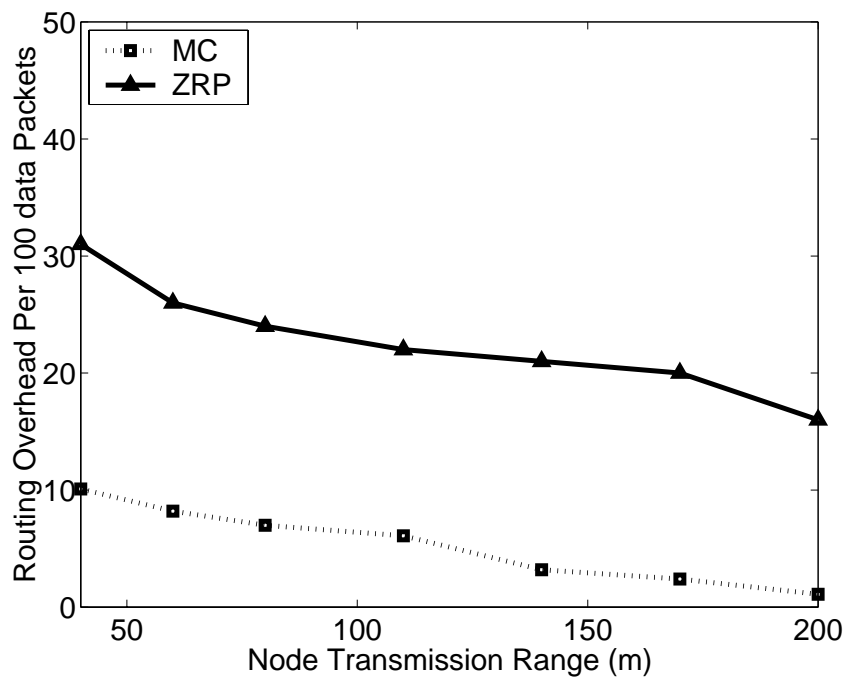

Fig. 3: Overhead Vs Tran. Range

\section{Throughput under Different Traffic Load}

We present the result of throughput comparison in Fig. 4. In the simulation, the maximum node speed is set to $20 \mathrm{~m} / \mathrm{s}$. Fig. 4 shows under ZRP, the network starts saturation when the traffic load is heavy. The throughput actually decreases when traffic load is larger than $250 \mathrm{kbits} / \mathrm{sec}$. This is because ZRP incurs large routing overhead from inter-zone routing and maintaining routes within zones. When the network traffic is heavy, congestion happens and packets are dropped in the network, and cause the throughput decreases.

\section{Delay Comparison}

The end-to-end delays for MC and ZRP under different traffic load are plotted in Fig. 5. As we can see, the delay of ZRP is close to MC when traffic load is light. However, when the traffic load becomes heavy, the delay of ZRP increases very fast, which means lots of congestion and packet drops are caused in the network. On the other hand, MC has much smaller routing overheads. There are little congestions and packet drops occur even under heavy traffic load. That is why the delay under MC routing increases slowly as the network traffic becomes heavy.

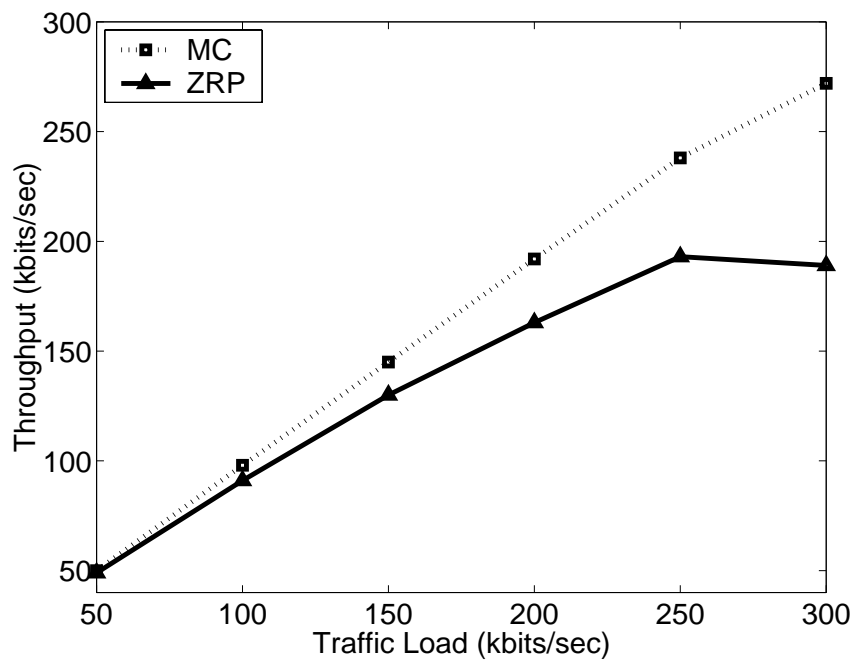

Fig. 4: Throughput Comparison

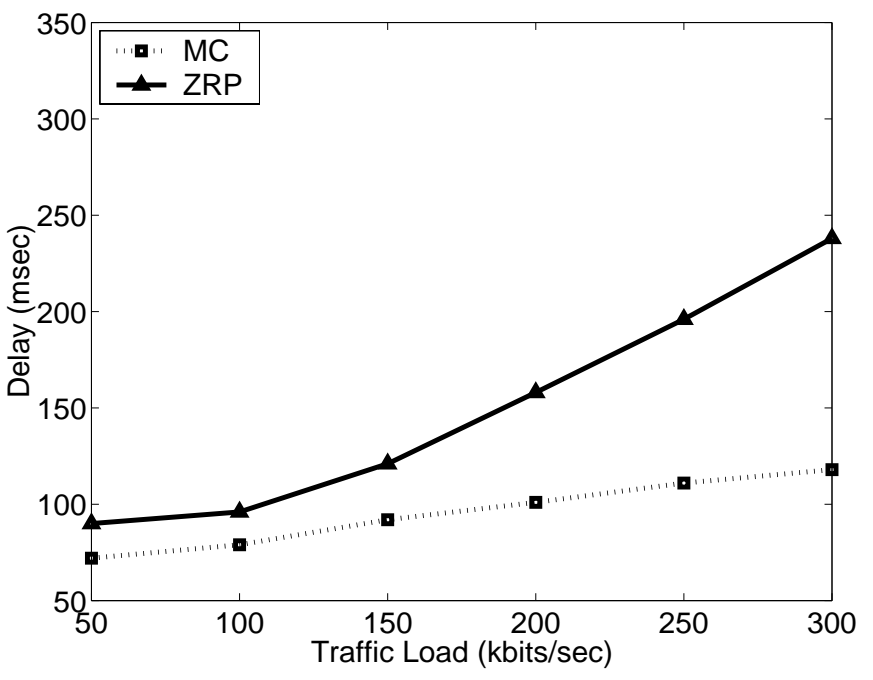

Fig. 5: Delay Comparison

\section{E. Scalability}

Scalability is a very important aspect of MANET routing protocol design. A good routing protocol should scale well in large networks. In order to test the scalability of MC, we implemented a large testbed with 400 nodes distributed uniformly at random in an area of $2000 \mathrm{~m} \times 2000 \mathrm{~m}$, where 100 nodes are Backbone-Capable nodes. The large testbed is four times the small testbed (in terms of node number and routing area) used in previous tests. We compare the performance of MC and ZRP in the large testbed. Fig. 6 reports the routing overheads of MC and ZRP for different mobility in large testbed, which are labeled as MC-L, ZRP-L. For comparison, in Fig. 6, we also plot the routing overheads of MC and ZRP in small testbed, which are labeled as MC-S, ZRP-S. Fig. 6 shows that the routing overhead of MC-L (in large testbed) is about twice as the overhead of MC-S, but MC-L is still small 
even under high mobility. However, the routing overhead of ZRP-L is much larger than ZRP-S, especially under high mobility. For the same source-destination pair, MC needs much less intermediate nodes than ZRP because MC uses Bnodes to route packets, which have long transmission range. So the chance of repairing or re-routing is much smaller in $\mathrm{MC}$ than in ZRP. Also MC has low routing latency as discussed in Subsection IV.A. Thus the routing overhead of $\mathrm{MC}$ in large network is still small.

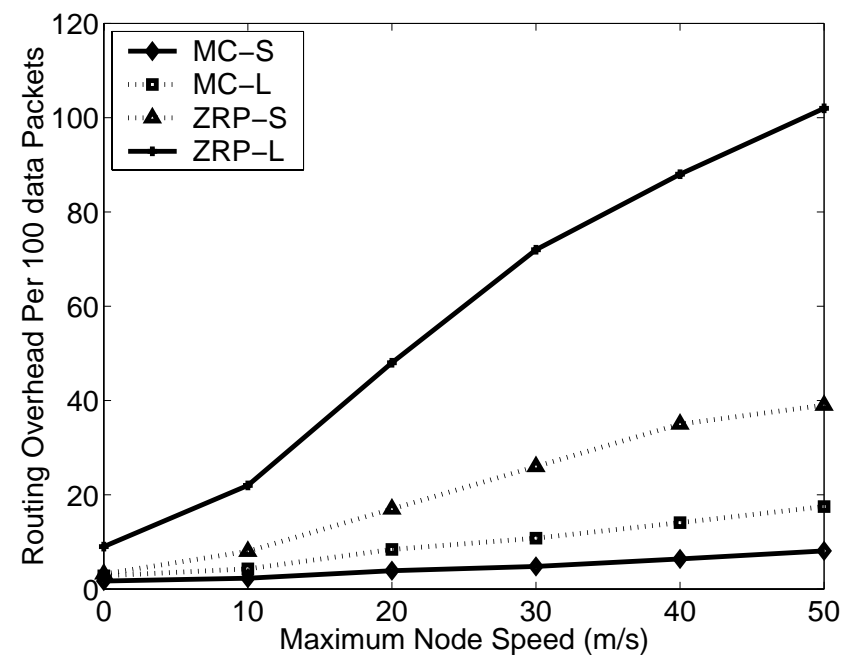

Fig. 6: Scalability - Routing Overhead

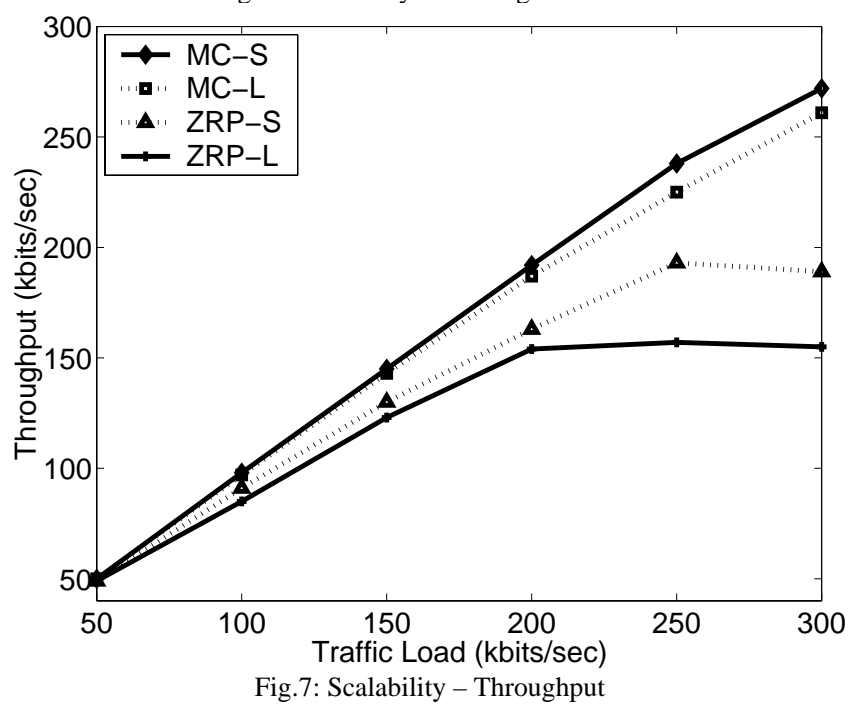

We also measured the throughputs of MC and ZRP in a large testbed, the results are reported in Fig. 7. For comparison, the throughputs of $\mathrm{MC}$ and ZRP in the small testbed are also plotted in Fig. 7. The labels have the same meaning as in Fig. 6. As we can see, the throughput for MC-L is almost the same as MC-S. However, the performance of ZRP becomes worse in large network than in small network. For the larger network, the throughput of ZRP-L drops when traffic load is larger than $200 \mathrm{kbits} / \mathrm{sec}$.

\section{F. The Performance of HMAC}

We studied the performance of the Hybrid MAC protocol. We compared the throughput and delay of MC routing using HMAC and IEEE 802.11b, and the results are reported in Figs. 8 and 9. Figs. 8 and 9 show that HMAC provides higher throughput and incurs smaller delay than IEEE 802.11b.

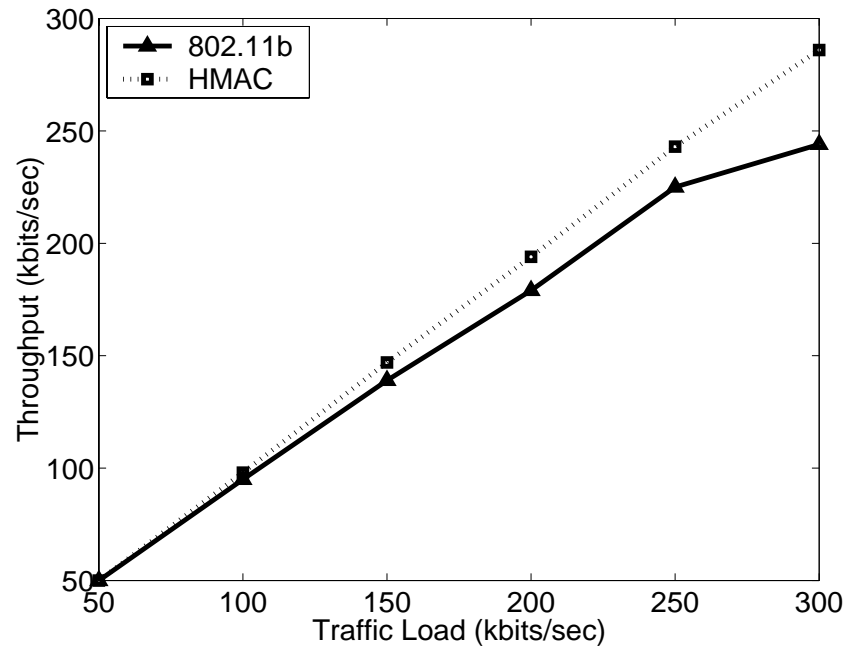

Fig. 8: The Throughput Performance

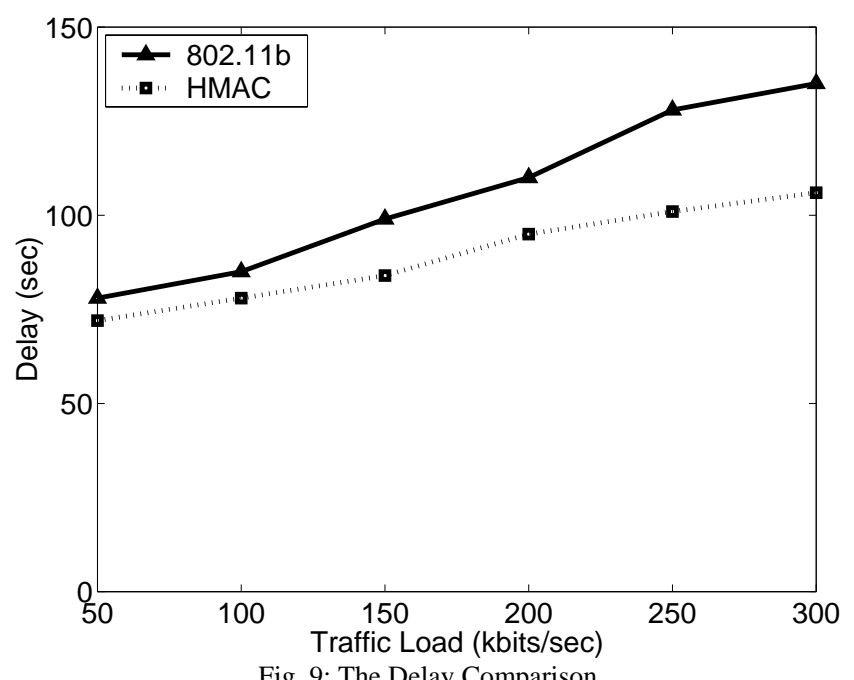

\section{G. Performance for Different BC-Node Densities}

To ensure the good performance of MC routing protocol, it is important to have B-node in most cells. If there is no Bnode in a cell, then B-node flooding (during route discovery) or small-area flooding (during route repair) may be used, which increase routing overhead. In [2], Du proved there is a high probability that each cell has at lease one B-node with a reasonable number of BC-nodes (about 1.5 times the cell number) uniformly and randomly distributed in the network.

We also use simulation to evaluate the performance of $\mathrm{MC}$ for different BC-node densities. In the simulation, the number of BC-nodes in MC varies from 5 to 35, with an increase of 5. We compare the routing overhead of MC and ZRP for the different densities of BC-nodes. The experiment results show that MC has larger routing overhead than ZRP when the 
density of BC-nodes is low (for less than $10 \mathrm{BC}$-nodes), but the routing overhead of MC decreases quickly as the number of BC-nodes increases.

\section{CONCLUSION}

This paper presents Multi-Class routing, a novel routing protocol that takes advantage of different communication capabilities of heterogeneous nodes in many realistic mobile ad hoc networks. An efficient MAC layer protocol - HMAC for heterogeneous MANETs is also presented. Different types of nodes have different transmission power (range), bandwidth, processing capability, reliability and security. MC routing utilizes the more powerful nodes as backbone nodes (B-nodes). The routing area is divided into several small, equal-sized cells. One B-node is maintained in each cell, and the routing among B-node is very efficient and simply based on location information and cell structure. A source discovers a route to destination in an on-demand way, and most of the routing activities (packet forwarding) are among B-nodes. This reduces the number of routing hops and makes the routing more efficient and reliable, since B-nodes have large bandwidth, transmission range and are more reliable. Extensive simulation tests demonstrate that MC has very good performance, and performs much better than ZRP in terms of reliability, scalability, routing overhead as well as packet delay and throughput.

\section{REFERENCE}

[1] Z. J. Haas, M. R. Pearlman, and P. Samar, "The Zone Routing Protocol (ZRP) for Ad Hoc Networks," Internet Draft, www.ietf.org/proceedings/02nov/I-D/draft-ietf-manet-zone-zrp-04.txt

[2] X. Du, "QoS Routing Based on Multi-Class Nodes for Mobile Ad Hoc Networks", Journal of Ad Hoc Networks, Vol. 2/3 pp 241-254, July 2004.

[3] Y.B. Ko and N. H. Vaidya, "Location-aided Routing (LAR) in Mobile Ad Hoc Networks," Proc. of ACM/IEEE MobiCom 1998, pp. 66-75.

[4] K. Xu, X. Hong, M. Gerla, "An Ad Hoc Network with Mobile Backbones,” Proc. of IEEE ICC 2002, New York, NY, Apr. 2002.

[5] B. Karp and H. T. Kung, "GPSR: Greedy Perimeter Stateless Routing for Wireless Networks," Proc. of ACM/IEEE MobiCom 2000.

[6] P. Sinha and S. Krishnamurthy, "Scalable Unidirectional Routing with Zone Routing Protocol (ZRP) Extenstions for Mobile Ad hoc Networks," Proc. of WCNC, Chicago, Sep. 2000.
[7] V. Ramasubramanian, et al., "Providing a Bidirectional Abstraction for Unidirectional Ad Hoc Networks,” Proc. of IEEE INFOCOM 2002.

[8] QualNet, Scalable Network Technologies, http://www.qualnet.com/.

[9] L. Bao and JJ Garcia-Luna-Aceves, "Link State Routing in Networks with Unidirectional Links," Proc. of IEEE ICCCN 1999, Boston, MA.

[10] S. Basagni et al., "A Distance Routing Effect Algorithm for Mobility (DREAM)," Proc. of ACM/IEEE MobiCom 1998, pp. 76-84.

[11] J. Camp, and L. Wilcox, Location Information Services in Mobile Ad Hoc Networks, Proc. of the IEEE ICC 2002.

\section{BIOGRAPHY}

Xiaojiang (James) Du (S'99--M'03) is an assistant professor in Dept. of Computer Science, North Dakota State University. Dr. Du received his B.E. degree from Tsinghua University, Beijing, China in 1996, and his M.S. and Ph.D. degrees from University of Maryland, College Park in 2002 and 2003, respectively, all in Electrical Engineering. His research interests are wireless sensor networks, mobile ad hoc networks, wireless networks, computer networks, network security and network management. He is a TPC member for several international conferences (including IEEE ICC 06, Globecom 05, BroadNets 05, WirelessCom 05, IPCCC 06 \& 05, and BroadWise 04). Dr. Du will serve as the program chair of Computer and Network Security Symposium of IEEE WirelessCom 2006.

Dapeng Wu (S'98--M'04) received Ph.D. in Electrical and Computer Engineering from Carnegie Mellon University, Pittsburgh, PA, in 2003. Since August 2003, he has been with Electrical and Computer Engineering Department at University of Florida as an Assistant Professor.

Wei Liu received the B.E. and M.E. degrees in Electrical Engineering from Huazhong University of Science and Technology, Wuhan, China, in 1998 and 2001, respectively. He is currently pursuing the $\mathrm{PhD}$ degree in the Department of Electrical and Computer Engineering, University of Florida, Gainesville, where he is a research assistant in the Wireless Networks Laboratory (WINET). His research interest includes QoS, Secure, Power Efficient Routing, MAC protocols in Mobile Ad Hoc Networks and Wireless Sensor Networks.

Dr. Fang received a Ph.D. degree in Systems Engineering from Case Western Reserve University in January 1994 and a Ph.D. degree in Electrical Engineering from Boston University in May 1997. He joined University of Florida in May 2000 as an assistant professor and has been a professor since August 2005. He has published over 150 papers in refereed professional journals and conferences. He received the National Science Foundation Faculty Early Career Award in 2001 and the Office of Naval Research Young Investigator Award in 2002. He has served on many editorial boards of technical journals including IEEE Transactions on Communications, IEEE Transactions on Wireless Communications, IEEE Transactions on Mobile Computing and ACM Wireless Networks. He is a senior member of the IEEE. 